\title{
The Performance of the ZEUS Central Tracking Detector $z$-by-Timing Electronics in a Transputer Based Data Acquisition System
}

\author{
B. Foster, G.P. Heath, T.J. Llewellyn \\ University of Bristol, H.H. Wills Physics Laboratory, Tyndall Avenue, Bristol BS8 1TL, England \\ D.M. Gingrich, N. Harnew, P.M. Hallam-Baker, T. Khatri, I.C. McArthur, P. Morawitz, J. Nash, \\ P.D. Shield, S. Topp-Jorgensen, F.F. Wilson \\ University of Oxford, Department of Nuclear Physics, Keble Road, Oxford OX1 3RH, England
}

D.B. Allen, R.C. Carter, M.D. Jeffs, M.C. Morrissey, S.P.H. Quinton

Rutherford Appleton Laboratory, Chilton Didcot, Oxon, OX11 0QX, England

\author{
J.B. Lane, M. Postranecky \\ University College, Department of Physics and Astronomy, Gower Street, London WC1E 6BT, England.
}

The Central Tracking Detector of the ZEUS experiment employs a time difference technique to measure the $z$ coordinate of each hit. The method provides fast, three-dimensional space point measurements which are used as input to all levels of the ZEUS trigger. Such a tracking trigger is essential in order to discriminate against events with vertices lying outside the nominal electron-proton interaction region. Since the beam crossing interval of the HERA collider is $96 \mathrm{~ns}$, all data must be pipelined through the front-end readout electronics. Subsequent data aquisition employs a novel technique which utilizes a network of approximately 120 INMOS transputers to process the data in parallel. The $z$-by-timing method and its data aquisition have been employed successfully in recording and reconstructing tracks from electron-proton interactions in ZEUS.

\section{Introduction.}

The Hadron Electron Ring Accelerator (HERA) at DESY is designed to collide $30 \mathrm{GeV}$ electrons on $820 \mathrm{GeV}$ protons at a beam crossing interval of $96 \mathrm{~ns}$. The ZEUS detector is one of two experiments investigating these collisions [1]. The Central Tracking Detector (CTD) is a $2 \mathrm{~m}$ long, $1.6 \mathrm{~m}$ diameter cylindrical drift chamber with a polar angular coverage of 15 to 164 degrees [2]. It is located at the heart of the ZEUS detector inside a uniform $1.43 \mathrm{~T}$ magnetic field. The CTD has 24192 wires, 4608 of which are sense wires, organised into nine radial superlayers. The five odd numbered superlayers lie parallel to the beam axis, while those of the even numbered layers are rotated at $5^{\circ}$ stereo angles. The superlayers are subdivided by field and shaper wires into "cells", tilted at $45^{\circ}$ with respect to the radius vector and each containing eight sense wires. The maximum drift time within a cell is $500 \mathrm{~ns}$. By measuring pulse heights and arrival times on the sense wires, the CTD accurately determines the momentum and charge of particles using a FADC system clocked at $104 \mathrm{MHz}$ [3]. This system measures $r-\phi$ drift distances to a precision of $130 \mu \mathrm{m}$ and ionisation loss to $6 \%$.

The CTD not only allows accurate reconstruction of charged particle tracks, but also contributes vitally to the trigger of ZEUS. When the full design luminosity of HERA is reached, the raw interaction rate in the ZEUS detector could be as high as $100 \mathrm{kHz}$. This rate is dominated by proton beam-gas interactions which swamp the $\sim 1 \mathrm{~Hz}$ of interesting physics events. Beam-gas events are expected to originate anywhere from up to $100 \mathrm{~m}$ upstream of the interaction point in the direction of the incident proton, whereas e-p collisions will occur only within the nominal interaction region defined by the intrinsic sizes of the colliding electron and proton bunches. The CTD can discriminate against these uninteresting background events by providing a coarse, but rapid, measurement of the event vertex in $z$ (the 
coordinate along the axis of the colliding beams). To achieve this, all sense wires in superlayer 1 and the four odd numbered sense wires in each of superlayers 3 and 5 are instrumented with $z$-bytiming electronics, making 704 channels in total. The principle of the method is to determine the $z$ position of a hit along a sense wire by measuring the time difference between the signals arriving at each end of the wire. To achieve the required vertex discrimination the time difference must be measured to an accuracy of $200 \mathrm{ps}$, corresponding to $3 \mathrm{~cm}$ in the $z$ coordinate $[4,5]$.

\section{The $z$ Readout Electronics.}

A schematic diagram of a $z$ readout channel is shown in Figure 1. On the passage of a charged track, a chamber pulse arrives at either end of the chamber where it is amplified by preamplifiers mounted on the CTD end flanges. The pulses then travel down $42 \mathrm{~m}$ of coaxial cable to postamplifiers, the total voltage gain being roughly 140 . The CTD readout is electronically divided into 16 sectors, each having an equal number of channels. Each single sector is read out by a rack of electronics containing three crates. The postamplifiers are contained in the first crate and here the chamber pulses are split and passed to the $z$-by-timing crate and to the $r-\phi$ FADC crate. A $z$-by-timing crate contains eleven $z$ cards (each with four channels per card) and seven or eight CTD First Level Trigger (FLT) cards.

At a $z$ readout card the chamber pulses are discriminated and input to a Time to Amplitude Converter (TAC) [4]. The "start" pulse from the rear end of the chamber starts the charging of a capacitor at constant current, which is then stopped by the "stop" pulse from the forward end of the chamber. The voltage level on the capacitor is therefore proportional to the time difference between the pulses, and hence to the $z$ coordinate of the hit. The voltage level is sampled by a FADC, digitised into 7 bits and latched together with an 8th bit validity flag.

In order to achieve the necessary performance of the $z$-by-timing system it is essential to maintain accurate calibration. The $z$ calibration system consists of a "Calibration Driver" module

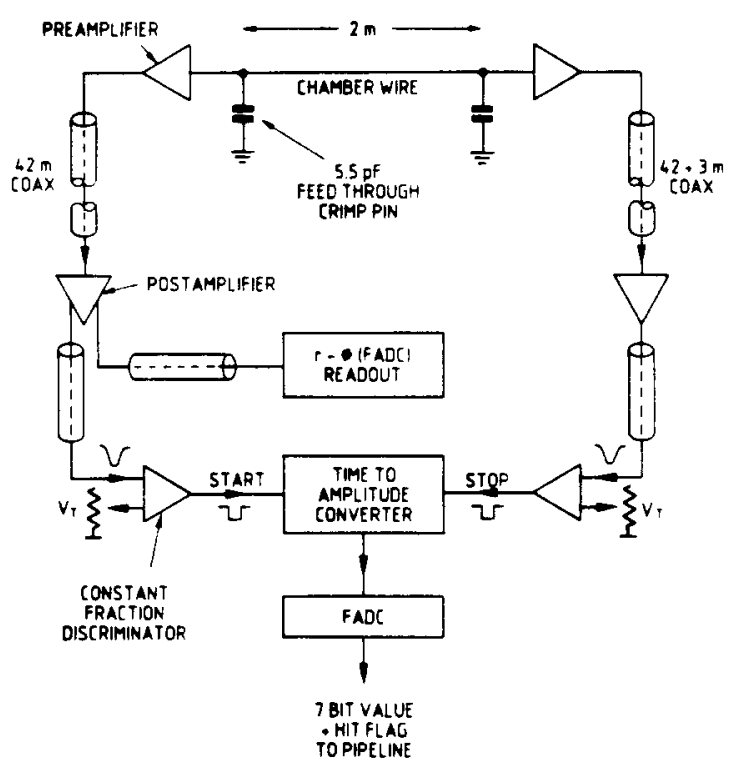

Figure 1. A block diagram of the front end $z$ readout from the chamber wire ends to the Time to Amplitude Converter (TAC).

which is mounted in each postamplifier crate and a "Calibration Controller" mounted in each $z$ crate. The Driver module sends calibration pulses onto the inputs of the preamplifiers generating shaped chamber-like pulses with risetimes of $2 \mathrm{~ns}$ very close to the wire ends. A channel is said to be calibrated when the full 7 bit dynamic range of the FADC corresponds to the full length of the chamber, ie. when the FADC values 0 and 127 represent the actual wire end points. Two parameters are available to effect this. Firstly a programmable $0-6 \mathrm{~ns}$ delay is used to vary the timing of the stop signal. Secondly the slope of the constant current ramp at which the capacitor is charged is controlled using a DAC. Thus, by pulsing alternate ends of the chamber, these parameters are varied until the returned $z$ values are 0 and 127 respectively. The Calibration Controller module supervises the calibration of a single crate and the complete calibration sequence is controlled via a single command to this module.

In addition to the calibration of the $z$-by-timing 
system, the calibration process is used as a general diagnostic tool to check the electrical continuity of every instrumented wire. Calibration can be performed both prior to a run and during a run using prescheduled test triggers arriving within empty beam buckets of the HERA machine.

Figure 2 shows how the $z$-by-timing readout is implemented within the local CTD data aquisition (DAQ) framework. The $z$ data is input to the CTD First Level Trigger (FLT), a pipelined hardware trigger which attempts to characterise an event in terms of the numbers of "good" and "bad" tracks with a determination of a valid event vertex where possible [6]. The CTD and other components then pass on their information to the Global First Level Trigger (GFLT) which then returns a trigger decision back to the components at a maximum average rate of $\sim 1 \mathrm{kHz}$. The total processing time involved in the FLT decision is $\sim 5 \mu \mathrm{s}$, hence it is necessary to store the $z$ event data for more than 50 beam crossings. To achieve this the 8-bit $z$ information is latched into a RAM pipeline. This is clocked at a $48 \mathrm{~ns}$ interval which is directly derived from the $96 \mathrm{~ns}$ beam crossing period. The $48 \mathrm{~ns}$ clock speed defines the multihit resolution of the $z$ system.

The pipeline and buffers of the front end electronics are driven by "Local Timing Controller" (LTC) cards, one in each of the $16 z$ crates. The system is synchronised to the HERA bunch crossings by a clock from the GFLT, passed via a single "Master Timing Controller" (MTC). The MTC sends the clock, control signals and trigger information from the GFLT to the LTCs and receives their busy signal. On receipt of a GFLT accept, the window of data in the $z$ pipeline containing the entire drift time for the corresponding event is transferred into a dual-port memory. This allows a maximum of 10 events to be buffered after a GFLT accept, necessary to cope with the varying instantaneous trigger rates. If no GFLT is received, the data in the pipeline are eventually overwritten by subsequent events. If any LTC is busy it means that the system is not ready for a subsequent trigger, hence the system incurs deadtime. The DAQ software is notified of buffered events which are awaiting readout via an interrupt register in the LTC.

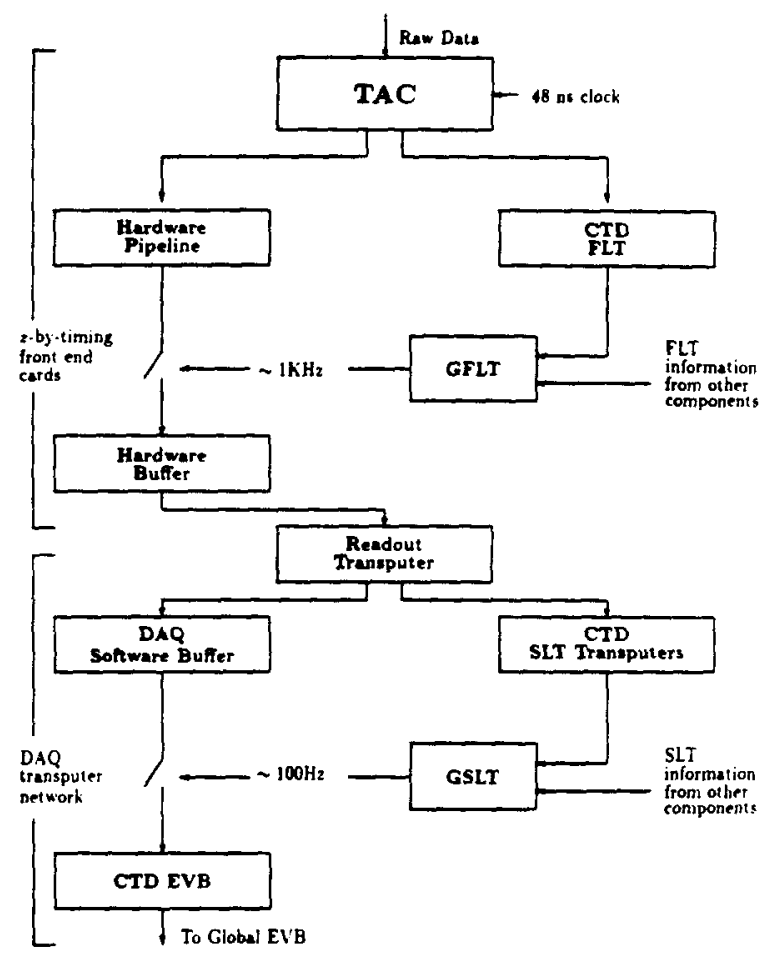

Figure 2. A block diagram showing the implementation of the $z$-by-timing information and its readout within the CTD DAQ system.

After a GFLT accept the data are zero suppressed, placed in software buffers and passed on to the CTD Second Level Trigger (SLT), a transputer based software trigger [7]. With the arrival of a corresponding Global Second Level Trigger (GSLT) accept decision (at a maximum average rate of $\sim 100 \mathrm{~Hz}$ ) the event data are transferred from the software buffers, concatenated, reformatted and passed, along with all other components' event data, to the ZEUS Event Builder (EVB). From here they pass to the Third Level Trigger (TLT) which is the first trigger where the entire event data from every participating component is available. The TLT (a Silicon Graphics processor farm) performs global event filtering which includes running track reconstruction software on the $z$-by-timing data. Accepted events are written to disk at a few Hertz. 

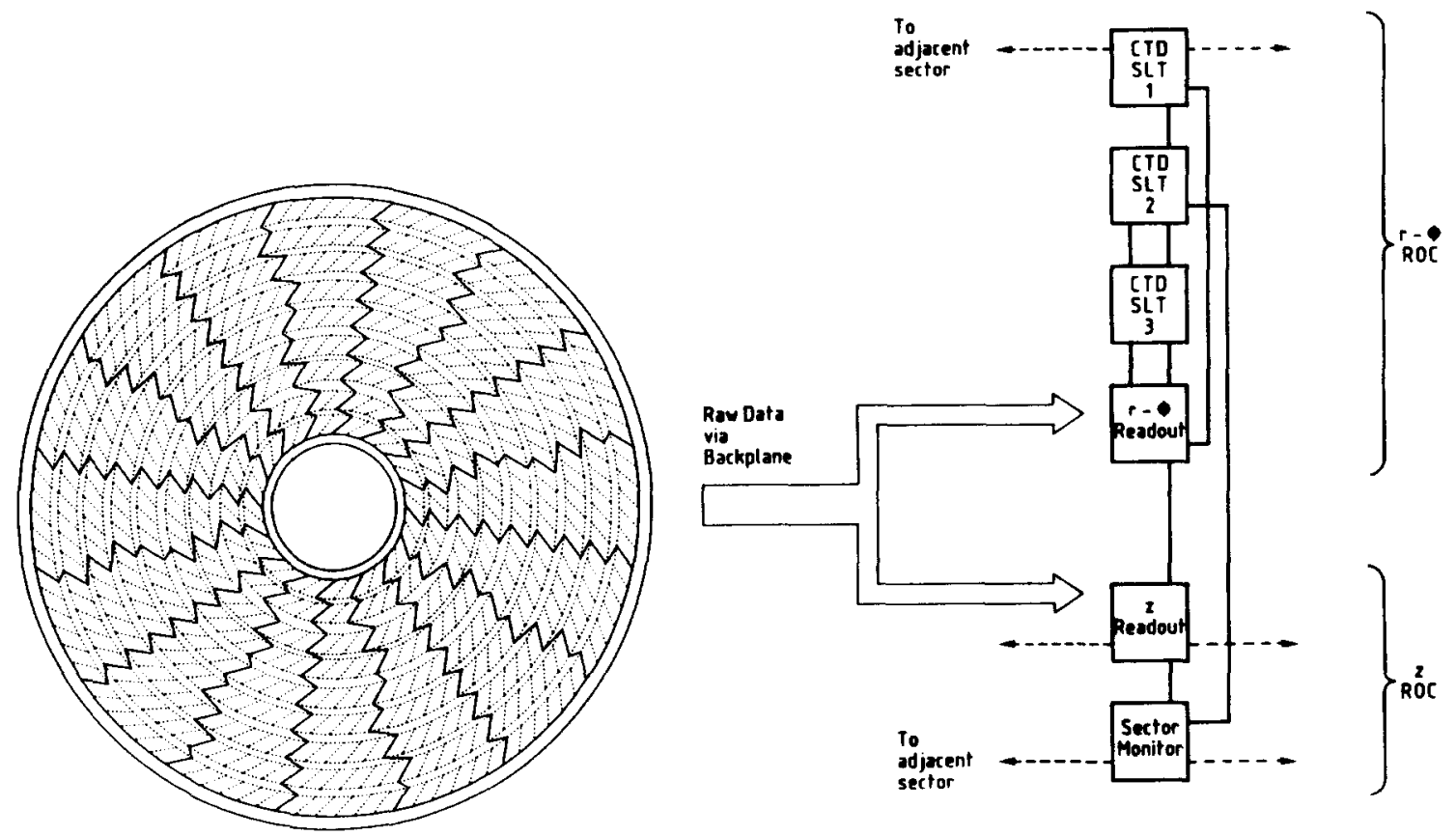

Figure 3. The geometrical symmetry of the CTD is exploited by allocating an identical block of 6 transputers to the readout of each of its 16 sectors. Each box represents a single transputer, connecting lines are communication links between them.

\section{The CTD DAQ System and its Imple- mentation.}

The CTD DAQ system is based on Transputer Readout Controllers (ROCs) containing two T425 transputers with 1 MByte (expandable to 4 MByte) of external memory [8]. One ROC controls the readout of a single crate. The T425 transputer is a single chip equipped with a 10 MIPS processor, on-chip memory, and four bidirectional $20 \mathrm{Mbit} / \mathrm{s}$ serial links. The processor unit and all four links are able to operate concurrently. The transputers are programmed in the language Occam which enables the coding of processes either in sequence or in parallel with channels carrying data packets of defined protocols between them [9]. Thus the transputer is ideal for constructing large reconfigurable DAQ processing networks. One transputer on the ROC is dedicated to readout and sees the backplane of the crate simply as an extension of its own memory and so is able to download parameters onto, and read data from, each of the front end cards. Extra external memory and processing power may be introduced as required by the addition of TRAM modules typically containing a T800 transputer plus 1 MByte of RAM. The T800 supports an additional on-chip floating point unit.

The natural way to handle the enormous data rates from HERA is to fully exploit the geometrical symmetry of the CTD in $\phi$ by treating each of its 16 sectors in parallel as far as possible. This is shown in Figure 3. Each of the $z$ and $r-\phi$ crates is read out by a ROC. In the $z$ ROC one transputer is dedicated to readout whilst the other performs data quality monitoring. The $r-\phi$ ROC has one readout transputer plus three CTD SLT transputers, of which two are T800s for floating point pattern recognition calculations. Between them these three transputers find track segments within individual cells and match segments between different superlayers to form tracks [7]. The 


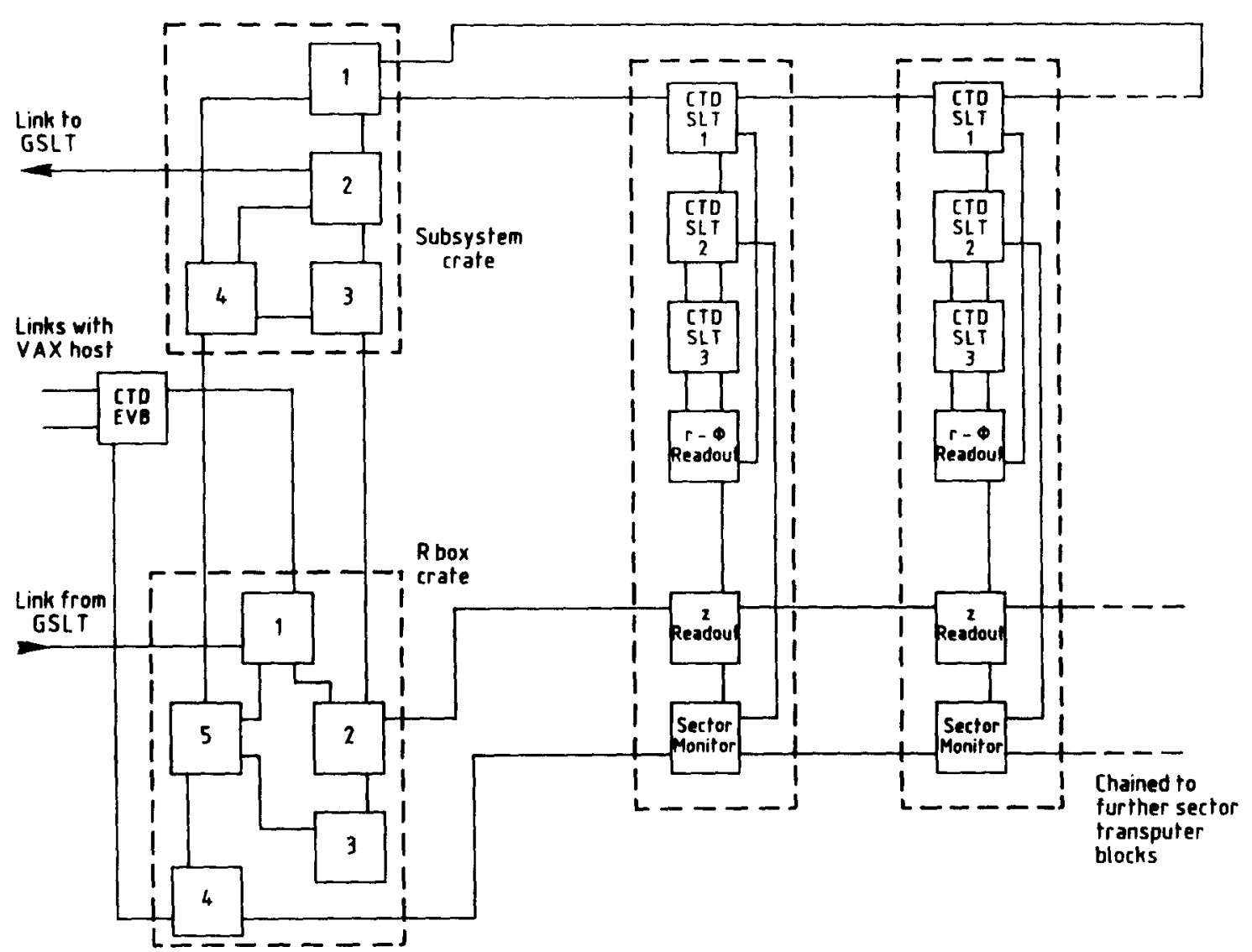

Figure 4. A representation of the CTD transputer DAQ network and its interconnections. The complete system is built from the chaining together of the transputer blocks of the 16 sectors, of which two are shown, plus the additional "Subsystem" crate and "RBox" crate transputers.

CTD SLT algorithm is optimised to find high $p_{T}$ tracks that originate in the vicinity of the nominal interaction point in the $r-\phi$ plane.

The inherent parallelism of the CTD data aquisition is also reflected in the code performing the readout and trigger tasks, which is identical for each sector. The modularity of the DAQ allows for simple extension as more electronics become available. Each new sector was introduced by replicating code and placing it appropriately on the transputers on each additional ROC.

Figure 4 shows the transputer network for two of the 16 sectors. Also shown are the networks in two additional crates, the "Subsystem" and the "RBox" crates, which complete the DAQ sys- tem. The Subsystem crate contains transputers which summarise the CTD SLT information from all sectors and then pass this information on to the transputer-based GSLT over a dedicated link. The RBox crate contains transputers which fan out the GSLT decision (also received on a dedicated link) to each of the 16 crate readout transputers. After a SLT accept, the data must be collected and passed to a transputer which interfaces directly via a triple-ported memory to the ZEUS Event Builder. The current network consists of 59 transputers which will eventually be expanded to 123 .

In order to force points of synchronization to this otherwise asynchronous DAQ system, the 
multitude of transputer tasks are rigidly defined within a set of hierarchically structured state managers. To facilitate the passing of messages between any pair of nodes in the transputer network, including the VAX host, a general purpose "harness" communication package has been developed with additional facilities for file and database serving and for event injection into the VAX buffer manager [10].

\section{The Performance of the $z$-by-Timing System at ZEUS.}

Extensive test-beam studies using a full length prototype of the CTD had been made prior to the installation of the CTD in ZEUS and the $3 \mathrm{~cm}$ design resolution for the $z$ position of a single hit has been realised [5]. Experience has now been gained of the $z$-by-timing and its DAQ system in the HERA environment. Results have been obtained during ZEUS cosmic ray and e$p$ collision runs under differing chamber operating conditions. Figure 5 shows the $z$ resolution as a function of chamber gas gain. Here the chamber was operating with a gas mix of 90:8:2 Argon: $\mathrm{CO}_{2}$ : Ethane with a $0.84 \%$ admixture of ethanol in a magnetic field of $1.43 \mathrm{~T}$ (under these conditions we have estimated that a sense wire surface field of $175 \mathrm{kV} / \mathrm{cm}$ corresponds approximately to a gas gain of $\left.1.0 \times 10^{5}\right)$. This shows that we have obtained a $z$ coordinate resolution of $(3.5 \pm 0.2) \mathrm{cm}$ in ZEUS, close to the design value. In addition, we measure preliminary hit efficiencies to be $(97 \pm 2) \%$. Reconstruction of tracks in the $r-\phi$ plane is possible using the $z$-by-timing system utilizing the coarse $48 \mathrm{~ns}$ sampling time. The measured $r-\phi$ resolution is $750 \mu \mathrm{m}$, close to the predicted value. One of the first e-p events recorded in the ZEUS detector is shown in Figure 6. This demonstrates the ability of the $z$-bytiming system to provide full three dimensional space points. Off-line reconstructed tracks have been superimposed on the figure and give good fits to the CTD hits. As the CTD will not be fully equipped with $r-\phi$ electronics until early 1993 , the $z$-by-timing system is providing the only tracking information available to the ZEUS experiment at this time.

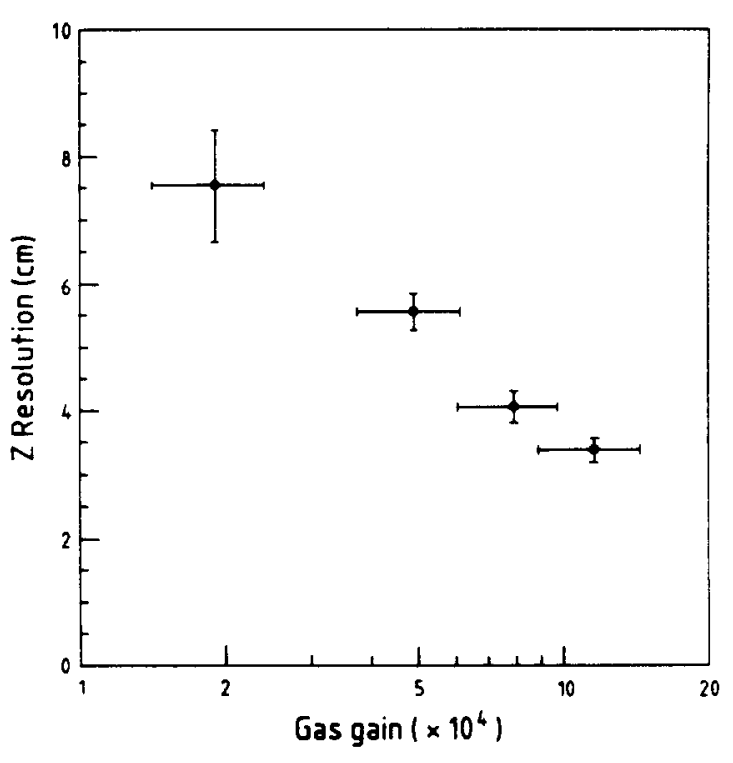

Figure 5. The resolution of the $z$ coordinate measurement as a function of chamber gas gain.

In summary, the CTD $z$-by-timing system has been integrated into the ZEUS DAQ framework, is working well, and is providing track reconstruction of $e-p$ collision data at HERA. We have achieved a $z$ coordinate resolution of $(3.5 \pm 0.2) \mathrm{cm}$ at a gas gain of $1 \times 10^{5}$.

We are grateful to R.Cranfield, G.J.Crone, D.A.Hayes, G.Nixon and S.F.Salih for technical, design and software effort associated with the Timing Controllers. We would also like to thank P.Chorley, R.Milborrow and S.Wood for development work associated with the $z$-by-timing readout modules. Finally, we are indebted to G.A.Blair for coordinating the smooth operation of the CTD at DESY. 

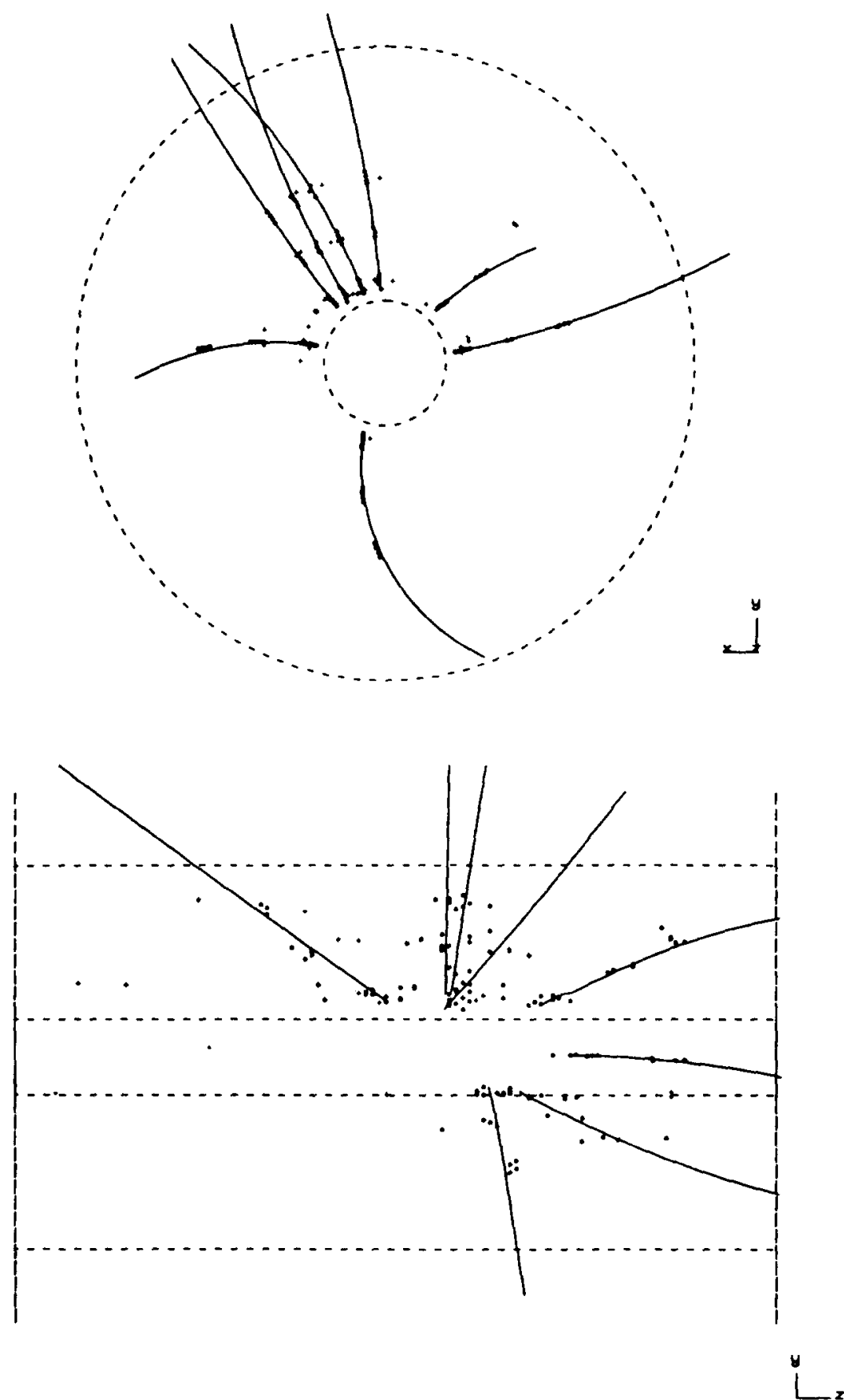

Figure 6. An e-p event recorded in the CTD with the $z$-by-timing system. Projections in $r-\phi$ and $r-z$ are shown, with offline reconstructed tracks superimposed on the raw hits. 


\section{REFERENCES}

1 ZEUS Collaboration, The ZEUS Detector Technical Proposal, 1986.

ZEUS Collaboration, The ZEUS Detector Status Report, 1989.

2 C.B.Brooks et al., Nucl. Inst. and Meth. A283 (1989) 477.

3 D.G.Cussans et al., Nucl. Inst. and Meth. A315 (1992) 397.

4 N.Harnew et al., Nucl. Inst. and Meth. A279 (1989) 290.

5 N.Harnew et al., Nucl. Inst. and Meth. A283 (1989) 781.

B.Foster et al., Proceedings of the 4th San Miniato Seminar on Experimental Apparatus for HEP and Astrophysics, World Scientific 1991, Eds. P.Giusti, F.L.Navarnia and P.G.Pelfer.

6 G.P.Heath et al., Nucl. Inst. and Meth. A315 (1992) 431.

7 R.C.E.Devenish et al., Proceedings of the CHEP90, Santa Fe, AIP Conference Proceedings 209 (1990) 155, Eds. J.Lillberg and M.Oothoudt.

8 S.P.H.Quinton et al., Proceedings of the IEEE 1989 Nuclear Science Symposium, San Francisco, IEEE Transactions on Nuclear Science 37 No. 6 (1990) 2161.

9 P.M.Hallam-Baker and I.C.McArthur, Computer Physics Communications 57 (1989) 520.

10 D.M.Gingrich et al., 2nd International Workshop on Software Engineering, Artificial Intelligence and Expert Systems for High Energy and Nuclear Physics, Jan 1992, to be published by World Scientific; University of Oxford preprint, OUNP-92-02. 